Methods We performed a multicenter retrospective analysis of consecutive acute ischemic stroke patients with M1 occlusion treated within 24 hours from the time of last known well who were greater than 89 years of age. Patient demographics, periprocedural metrics, and discharge and 90-day modified Rankin Scale (mRS) scores were collected. Successful recanalization was defined as TICI 2B or better. Favorable outcome was defined as 90-day mRS£2 for patients prestroke $m R S<2$, and no worsening of prestroke mRS at 90-day in patients with prestrike $\mathrm{mRS}>2$.

Results Seventy-one patients over 89 years old were identified. Fifty-two (73.2\%) were female. Pre-stroke mRS scores were available for 70 patients. The baseline median mRS was 2 . Successful recanalization was achieved in $95.8 \%$ of patients. TICI 2C or greater was accomplished in $64.8 \%$ of cases. Functional outcome data at 90-days were available for 57 patients $(80.2 \%)$. At three months, 7 total patients had an mRS between 0-2 (12.3\%), and an additional 11 patients had an $\mathrm{mRS}$ of 3 (19.2\%). The rate of 90-day mortality was $42 \%$.

Conclusions Mechanical thrombectomy in nonagenarians is technically feasible with high rate of successful reperfusion and it may improve clinical outcome. More research is needed to determine if thrombectomy results in significantly better functional outcomes than medical management for nonagenarians.

Disclosures S. Majidi: None. J. Vargas: 2; C; Cerenovus, Medtronic. 4; C; Truvic. J. Blalock: None. H. Hawk: None. S. Nimjee: None. A. Zakeri: None. M. Mokin: 2; C; Medtronic, Cerenovus. 4; C; Endostream, Serenity medical. R. Kellogg: None. G. Cortez: None. A. Aghaebrahim: None. E. Sauvageau: None. R. Hanel: 1; C; Stryker, Medtronic. 2; C; Stryker, Medtronic, Balt, Cerenovus, Microvention, Q'Apel, Rapid Medical. 4; C; Scientia, RisT, Corindus. R. DeLeacy: None. A. Siddiqui: 1; C; NIHNINDS R21 NS109575-01. 2; C; Cerenovus, Imperitive Care, Medtronic, Microvention, Penumbra, Q'Apel. 4; C; Imperitive Care, Q'Apel, Rist, Truvic. A. Turk: 2; C; Imperitive Care, Stryker, Microvention, Penumbra, BALT, Cerenovus. 4; C; Imperitive Care. M. Oselkin: None. E. Marlin: None. R. Turner: 2; C; Q'apel, Cerenovus, Siemens. 4; C; Q'APel. I. Chaudry: 2; C; Cerenovus, Q’Apel. 4; C; Q'Apel. J. Milburn: None.

\section{E-020 A NOVEL TECHNIQUE TO TREAT TANDEM OCCLUSION BY UTILIZING STENTRIEVER AS THE DISTAL PROTECTION DEVICE WITH ASPIRATION CATHETER}

${ }^{1} S$ Suzuki*, 'J Granstein, ${ }^{1} \mathrm{~A}$ Chan, ${ }^{1} \mathrm{~B}$ Ball, ${ }^{2} \mathrm{~V}$ Vu, ${ }^{1} \mathrm{~K}$ Golshani, ${ }^{1} \mathrm{I}$ Yuki. ${ }^{1}$ Neurological Surgery, Univiersity of California Irvine, Orange, $C A ;{ }^{2}$ Radiological Sciences, Univiersity of California Invine, Orange, CA

\subsection{6/neurintsurg-2021-SNIS.116}

Background Revascularization in acute ischemic stroke (AIS) with tandem occlusion (TO) is technically challenging due to the involvement of two large vessel occlusions (LVO) in the same vascular territory, usually with a large clot burden. There are two traditional approaches to re-establish the blood flow from TO; initial intracranial mechanical thrombectomy (MT) (head-first) or initial angioplasty \pm stenting (neck-first). We developed a novel technique to simultaneously revascularize distal and proximal occlusions. This is accomplished by utilizing a stent-retriever as a distal protection device and a large inner diameter aspiration catheter with a balloon guiding catheter to retrieve clot and prevent distal migration (flow-arrest proximal protection).

Subject and Methods Since January of 2018, we performed MT for 142 LVO in AIS of the anterior circulation, of which we treated 12 TO. For the last six cases of TO, the Novel technique was applied to revascularize and restore perfusion. Patient demographics, procedure time (artery puncture to closure), procedure safety including symptomatic intracranial hemorrhage (sICH), angiographic outcomes (TICI score), and clinical outcomes (mRS) were evaluated.

Results The novel technique procedure in detail: an 8-9French balloon-guided catheter is advanced to the distal common carotid artery and the balloon is inflated for flow arrest proximal protection. A microcatheter and microguidewire are passed through the steno-occlusive site of the proximal internal carotid artery (ICA) and advanced into the M1 segment. After removing the micro-guidewire, a stentretriever is navigated into the microcatheter and deployed at the distal M1 occlusion. Subsequently, the microcatheter is removed. The stent-retriever promotes flow restoration and captures distal emboli. During several minutes of flow restoration, a balloon angioplasty catheter is navigated to the proximal ICA steno-occlusive site over a stent-retriever wire, and balloon angioplasty is performed. After balloon angioplasty catheter removal, an aspiration catheter is advanced into the internal carotid artery, followed by the M1 segment, over the stent-retriever wire. Clot aspiration, retrieval, and capture of the stent-retriever is then performed. Carotid stenting is not used due to concerns for intracranial hemorrhage related to antiplatelet therapy in the acute phase. Among six patients, the mean age was 68 yo (41 - 83), median NIHSS at presentation was 18 (5 $24)$, sICH was $1 / 6$, and mortality was $1 / 6$. One patient died from concomitant COVID-19 pneumonia despite successful revascularization and neurological improvement. TICI IIb or III was achieved in 5/6 patients, and mRS 0-2 was observed in $3 / 6$ patients. The mean procedure time was 122 minutes (89 - 157), which was shorter than the TO group treated with traditional approaches 144 minutes (95 - 184), though this was not statistically significant $(p=0.05$ t-test). However, with improved experience, the procedure time decreased, and the last two cases' procedure times were 89 and 91 minutes respectively.

Conclusion The novel technique to revascularize tandem occlusion utilizing a stent-retriever as a distal protection device along with an aspiration catheter and a balloon-guided catheter is feasible. Immediate flow restoration at the distal occlusion site with simultaneous revascularization of the proximal ICA steno-occlusive lesion potentially improves the puncture to reperfusion time.

Disclosures S. Suzuki: None. J. Granstein: None. A. Chan: None. B. Ball: None. V. Vu: None. K. Golshani: None. I. Yuki: None. 